Research Paper

\title{
Clinical diagnostic value of long non-coding RNAs in Colorectal Cancer: A systematic review and meta-analysis
}

\author{
Bi Chen ${ }^{1,2,3,4}$, Ruo Nan Zhang, 1,2,3, , Xingxing Fan ${ }^{1,2}$, Jue Wang1,2, Cong $\mathrm{Xu}^{1,2}$, Bo An ${ }^{1}$, Qiao Wang1, Jing \\ Wang ${ }^{1}$, Elaine Lai-Han Leung ${ }^{1,2}$, Xinbing Sui ${ }^{3,4}$ and Qibiao $\mathrm{Wu}^{1,2 \bowtie}$ \\ 1. Faculty of Chinese Medicine, Macau University of Science and Technology, Taipa, Macau, P.R. China. \\ 2. State Key Laboratory of Quality Research in Chinese Medicines, (Macau University of Science and Technology), Taipa, Macau, P. R. China. \\ 3. Department of Medical Oncology, Holistic Integrative Oncology Institutes and Holistic Integrative Cancer Center of Traditional Chinese and Western \\ Medicine, the Affiliated Hospital of Hangzhou Normal University, College of Medicine, Hangzhou Normal University, Hangzhou, Zhejiang, China. \\ 4. Department of Cancer Pharmacology, Holistic Integrative Pharmacy Institutes, College of Medicine, Hangzhou Normal University, Hangzhou, Zhejiang, \\ China. \\ $\triangle$ Corresponding authors: E-mails: lhleung@must.edu.mo (E. Leung); hzzju@hznu.edu.cn (X. Sui); qbwu@must.edu.mo (Q. Wu).
}

() The author(s). This is an open access article distributed under the terms of the Creative Commons Attribution License (https://creativecommons.org/licenses/by/4.0/). See http://ivyspring.com/terms for full terms and conditions.

Received: 2020.03.26; Accepted: 2020.06.24; Published: 2020.07.11

\begin{abstract}
Background: Histopathological diagnosis remains the gold standard for the diagnosis of cancer, including colorectal cancer, but it is infeasible when tumor tissue is not available. With the recognition of long non-coding RNAs (IncRNAs), the expression of IncRNAs in serum or tissue samples has been reported as a diagnosis method for some cancers, however, the diagnostic value of IncRNAs for colorectal cancer remains unclear.

Methods: A systematic review and meta-analysis were conducted. Eligible studies were identified through a comprehensive literature search in PubMed, PubMed Central, Web of Science, Embase, and Cochrane Library (up to May 05, 2020) according to the selection criteria. Meta-DiSc, Review Manager and STATA were used to analyze the association between IncRNAs expression and the diagnosis of colorectal cancer.

Results: Fifteen studies that analyzed the expression of 15 IncRNAs in 1434 CRC patients were included. The summary area under the curve (AUC) of IncRNA for the diagnosis efficacy between patients with and without CRC was estimated to be 0.8629 , corresponding to a weighted sensitivity of 0.75 ( $95 \% \mathrm{Cl}: 0.72-0.77$ ), specificity of 0.80 ( $95 \% \mathrm{Cl}: 0.78-0.82)$. Subgroup analysis illustrated that the AUC of blood-based detection of IncRNA showed 0.8820, pooled DOR: 18.57, while tissue-based analysis showed 0.8203, pooled DOR: 10.47. Blood-based tests were then divided into two categories, plasma-based and serum-based IncRNA testing. Results revealed that the AUC of serum-based detection was 0.9077 , pooled DOR: 26.64 , and plasma-based detection was 0.5000 , pooled DOR: 11.80 .

Conclusions: This meta-analysis indicates that the aberrantly expressed IncRNAs might serve as potential diagnostic biomarkers for CRC patients and blood-based IncRNA analysis is of higher diagnostic accuracy than tissue-based testing. Moreover, serum-based IncRNA testing achieved higher diagnostic efficacy than plasma-based analysis.
\end{abstract}

Key words: long non-coding RNAs; colorectal cancer; diagnosis; systematic review

\section{Introduction}

Based on the GLOBOCAN2018 evaluation criteria for cancer morbidity and mortality by IARC, colorectal cancer $(\mathrm{CRC})$ is still the third most commonly diagnosed malignancy with the second most lethal cancer rate worldwide [1]. According to the National Cancer Statistics released by the National Cancer Center in 2019, the incidence of colorectal cancers (CRC) ranks fourth among malignant tumors and has become the fifth leading cause of cancer mortality in China [2]. Previous studies have indicated that the colorectal cancer patients in the early stage would gain longer survival time with standard treatment [3-5]. The prognosis of CRC can be improved when the patients are identified at their 
early stages. Therefore, we are supposed to improve CRC diagnostic strategies, which is as important as discovering new treatments.

Currently, the main biomarkers of colorectal cancer in clinical practice consist of carcinoembryonic antigen (CEA), cancer antigen 19-9 (CA19-9) and cancer antigen 242 (CA242). But the sensitivity and specificity are far from satisfying clinical needs. A previous study has demonstrated that the sensitivity of CEA was $46.59 \%$ and its specificity was $80 \%$ [6]. Another study using ROC curves to comparing the specificity and sensitivity of CEA and CA19-9 in the early diagnosis of CRC, the AUC of CEA is 0.797 (P < $0.001)$ and the AUC of CA19-9 is $0.664(\mathrm{P}=0.001)$ [7]. There is a pressing need for more ideal biomarkers with higher sensitivity and specificity for early diagnosis of CRC.

Long non-coding RNAs (lncRNAs) are defined as capped transcripts $>200$ nucleotides [8]. It is reported that several lncRNAs are aberrantly expressed in tissue or serum from colorectal cancer patients. Some studies have reported that MEG3 [9], lnc-ATB [10] and BLACAT1 [11] were up-expressed in colorectal cancer plasma, while NKILA [7] and HOTAIRM1 [12] were down-regulated in colorectal cancer tissue. A sensitivity of $37.16 \%$ and a specificity of $88.75 \%$ for CEA were significantly lower than that for exosomal CRNDE-h $(P<0.001)$ [13]. NKILA exhibited relatively higher sensitivity and specificity compared with CEA and CA19-9 in the early diagnosis of CRC [7]. And LINC02418 was also more sensitive than those existing detection methods [14].

Studies based on LncRNAs detection are constantly springing up; however, the diagnostic value of lncRNAs for colorectal cancer remains unclear. So we performed a meta-analysis to evaluate the relationship between clinical outcomes (clinicopathological parameters, diagnosis) and the expression of lncRNAs in patients with colorectal cancer, hoping to provide a theoretical basis for clinical application.

\section{Materials and Methods}

\section{Literature Search}

Two of the authors (Bi Chen and Ruonan Zhang) each searched several databases, including PubMed, PubMed Central (PMC), Web of Science, Embase, and Cochrane Library. The publication data used in the literature search was from database inception to May 05, 2020. The search strategies were based on combinations of the following keywords in titles or abstracts: (lncRNA OR long ncRNA OR lincRNA OR long non-coding RNA OR long non-translated RNA OR long untranslated RNA OR long non-protein- coding RNA OR long intergenic non-protein coding RNA) and (colorectal cancer OR colorectal neoplasm OR colorectal tumor OR colorectal carcinoma) and ("diagnose" or "diagnosis").

\section{Selection Criteria}

All included studies met the following inclusion criteria: 1) patients were diagnosed with CRC by histopathology and without other tumors; 2) volunteers were defined as no tumor and other diseases; 3) the expression level of lncRNAs was identified and analyzed for the diagnosis of $\mathrm{CRC}$, and; 4) studies contained sufficient data, including sensitivity (SEN), specificity (SPE), area under the curve (AUC) or receiver operating characteristic (ROC). If unavailable, related data obtained by contacting the corresponding authors.

Exclusion criteria were: 1) duplicated study; 2) conference abstract, review, meta-analysis, animal or cellular study; 3) unrelated to CRC or lncRNA; 4) incomplete or uncorrected study.

\section{Data Extraction}

Two independent authors (Bi Chen and Ruonan Zhang) collected the following information from the literature for each publication: (1) basic information: first author, publication year, type of lncRNA, expression, detection sample, test method, cut-off value, number of patients; (2) clinicopathological information: $P$ values of age, gender, tumor location, tumor size, differentiation, lymphatic metastasis, distal metastasis, TNM stage, CEA level, CA 19-9 level and depth of invasion; (3) diagnosis information: SEN, SPE, AUC, ROC; (4) Engauge Digitizer software was utilized to obtain the SEN and SPE from ROC when data was not reported directly.

\section{Quality Assessment}

Study quality was assessed with the Quality Assessment of Diagnostic Accuracy Studies II (QUADAS-2) checklist, which is recommended for use in systematic reviews and applicability of primary diagnostic accuracy studies, consisting of four key domains covering patient selection, index test, reference standard, flow and timing. Each is assessed in terms of risk of bias and the first three in terms of concerns regarding applicability [15].

\section{Statistical Analysis}

The Meta-DiSc software (Version 1.4) was used to calculate the combined sensitivity, combined specificity, positive likelihood ratio (PLR), negative likelihood ratio (NLR), overall diagnostic odds ratio (DOR), diagnostic advantage and 95\% confidence interval for each study [16]. Review Manager (Version 5.3. Copenhagen: The Nordic Cochrane Centre, The 
Cochrane Collaboration, 2014) and STATA 15.0 software (StataCorp, College Station, TX 77845, USA) were used to analyze study data and construct the forest plot. Heterogeneity among studies was assessed by the Cochran's Q and the $I^{2}$ statistic. The fixed-effects model was used if there was no substantial heterogeneity $\left(P>0.10\right.$ or $\left.I^{2}<50 \%\right)$. Inversely, the random-effects model was chosen when significant heterogeneity was observed [17-19]. Associations between lncRNA expression and clinicopathologic parameters were determined using the $P$ values combined with Fisher's test. Publication bias was quantitatively judged by Deeks' funnel plot asymmetry test [20]. $P<0.05$ was considered to be statistically significant.

\section{Results}

\section{Study Selection}

We searched and captured 200 records in PubMed, PMC, EMBASE, Web of Science, and the Cochrane Library. Of these, 52 duplicate studies were excluded. We excluded 123 records after reading the titles and abstracts. Subsequently, we assessed the remaining 25 full-text articles and excluded 10 studies based on the exclusion criteria, including 4 reviews \& updates, 5 lacking key data, and 1 uncorrected proof version. A total of 15 studies were ultimately included in this study. A flow diagram of the selection process for this study is presented in Figure 1.

\section{Study parameters and study quality}

Eighty-five percent of the selected studies were from China, with 11/15 (73.3\%) being published between 2018 and 2020. A total of 1434 CRC patients were included and the number of patients ranged from 34 to 174. All patients were diagnosed based on the histopathological diagnostic criteria. The tissue samples and blood samples (serum and plasma samples) were obtained prior to clinical treatment. The expression levels of LncRNAs were determined using quantitative real-time polymerase chain reaction (qRT-PCR). Seven types of lncRNAs were recognized as tumor promoters [10, 11, 13, 14, 21-25] and six were tumor suppressors $[7,9,26-29]$ in CRC patients. The associations between lncRNAs and clinicopathologic parameters in CRC patients are shown in Table 1. Diagnostic accuracy differed greatly between different lncRNAs. We found that ENST00000455974 detection had the highest sensitivity (95.6\%) with a specificity of $81.2 \%$ [23] and CRNDE-h detection had the highest specificity $(91.5 \%)$ with a sensitivity of $70.3 \%$ [13].

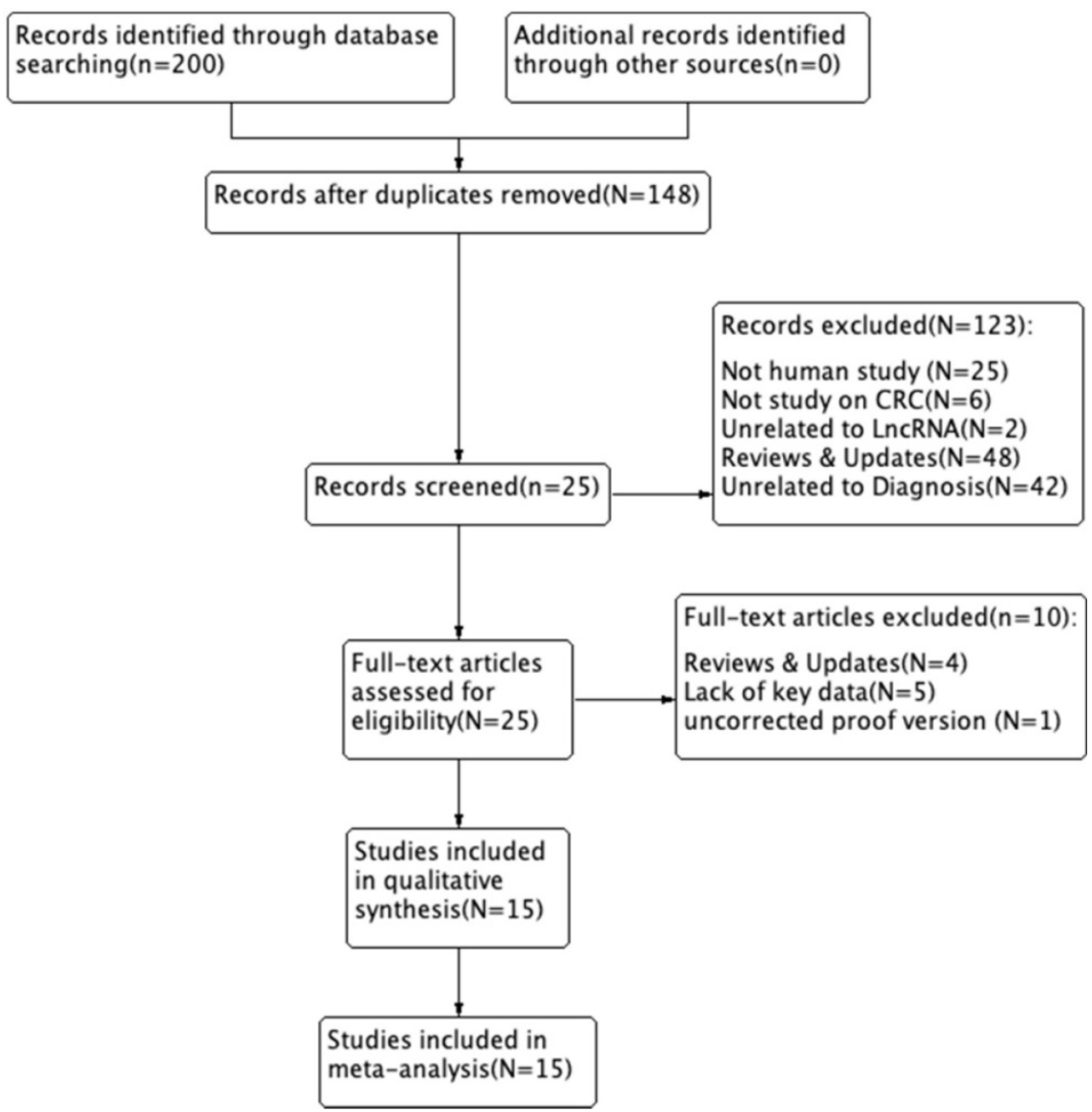

Figure 1. Flow diagram of the study selection process. 
Table 1. Main characteristics of the meta-analysis for diagnostic performance and clinicopathologic association of IncRNAs in CRC patients

\begin{tabular}{|c|c|c|c|c|c|c|c|c|c|c|c|c|c|}
\hline First author & $\begin{array}{l}\text { Publication } \\
\text { Year }\end{array}$ & LncRNA & Expression & $\begin{array}{l}\text { Patient } \\
\text { number }\end{array}$ & $\begin{array}{l}\text { Control } \\
\text { number }\end{array}$ & $\begin{array}{l}\text { Sample } \\
\text { size }\end{array}$ & $\begin{array}{l}\text { Sample } \\
\text { type }\end{array}$ & $\begin{array}{l}\text { Detection } \\
\text { method }\end{array}$ & $\begin{array}{l}\text { Cut-Off } \\
\text { Value }\end{array}$ & AUC & $95 \% \mathrm{CI}$ & SEN \% & SPE $\%$ \\
\hline Meng Xu & 2020 & HANR & up & 165 & 165 & 330 & tissue & qRT-PCR & meidan & 0.82 & $0.775-0.865$ & 60.0 & 82.0 \\
\hline Xianjuan Shen & 2020 & DANCR & up & 40 & 40 & 80 & serum & qRT-PCR & 1.994 & 0.747 & $0.638-0.857$ & 67.5 & 82.5 \\
\hline Baolian Song & 2019 & lnc RNA-1 & up & 77 & 30 & 107 & tissue & qRT-PCR & NA & 0.788 & NA & 83.7 & 64.2 \\
\hline Paria Abedini & 2019 & $\operatorname{lnc}-\mathrm{ATB}$ & up & 74 & 74 & 148 & plasma & qRT-PCR & 2.5000 & 0.780 & $0.811-0.940$ & 82.0 & 75.0 \\
\hline Peng Jiang & 2019 & NKILA & down & 70 & 140 & 210 & tissues & qRT-PCR & 4.5000 & 0.839 & NA & 82.9 & 72.9 \\
\hline Wei Wang & 2019 & MEG3 & down & 126 & 48 & 174 & serum & qRT-PCR & NA & 0.798 & $0.730-0.866$ & 66.7 & 87.5 \\
\hline Yinghui Zhao & 2019 & LINC02418 & up & 125 & 125 & 250 & serum & qRT-PCR & 2.9590 & 0.898 & $0.864-0.935$ & 95.2 & 66.4 \\
\hline Q.-G. LI & 2019 & lnc-DILC & down & 174 & 174 & 348 & tissue & RT-qPCR & median & 0.826 & NA & 78.0 & 71.0 \\
\hline Yueqiong Lao & 2018 & ENST00000455974 & up & 45 & 66 & 111 & tissues & qRT-PCR & 0.0005 & 0.899 & $0.821-0.977$ & 95.6 & 81.2 \\
\hline Jiangtao $\mathrm{Yu}$ & 2018 & SLCO4A1-AS1 & up & 50 & 15 & 65 & tissue & qRT-PCR & median & 0.924 & $0.852-0.996$ & 92.2 & 87.0 \\
\hline Yeshuo Ma & 2018 & RP1-85F18.6 & up & 34 & 34 & 68 & tissue & RT-qPCR & median & 0.651 & $0.516-0.785$ & 55.9 & 76.5 \\
\hline Nehal Samir & 2017 & LncRNA-RP11-909B2 & down & 70 & 60 & 130 & serum & qRT-PCR & 0,1800 & 0.867 & $0.807-0.925$ & 80.0 & 93.3 \\
\hline Ledong Wan & 2016 & HOTAIRM1 & down & 150 & 101 & 251 & plasma & qRT-PCR & 0.0030 & 0.780 & $0.708-0.841$ & 64.0 & 76.5 \\
\hline Tong Liu & 2016 & CRNDE-h & up & 148 & 320 & 468 & serum & RT-qPCR & 0.0200 & 0.892 & $0.860-0.918$ & 70.3 & 94.4 \\
\hline Debing Shi & 2014 & RP11-462C24.1 & down & 86 & 86 & 172 & tissue & qRT-PCR & mean & 0.778 & NA & 69.4 & 73.9 \\
\hline
\end{tabular}

LncRNA: long non-coding RNA; AUC: area under the curve; SEN: sensitivity; SPE: specificity; NA: not available.

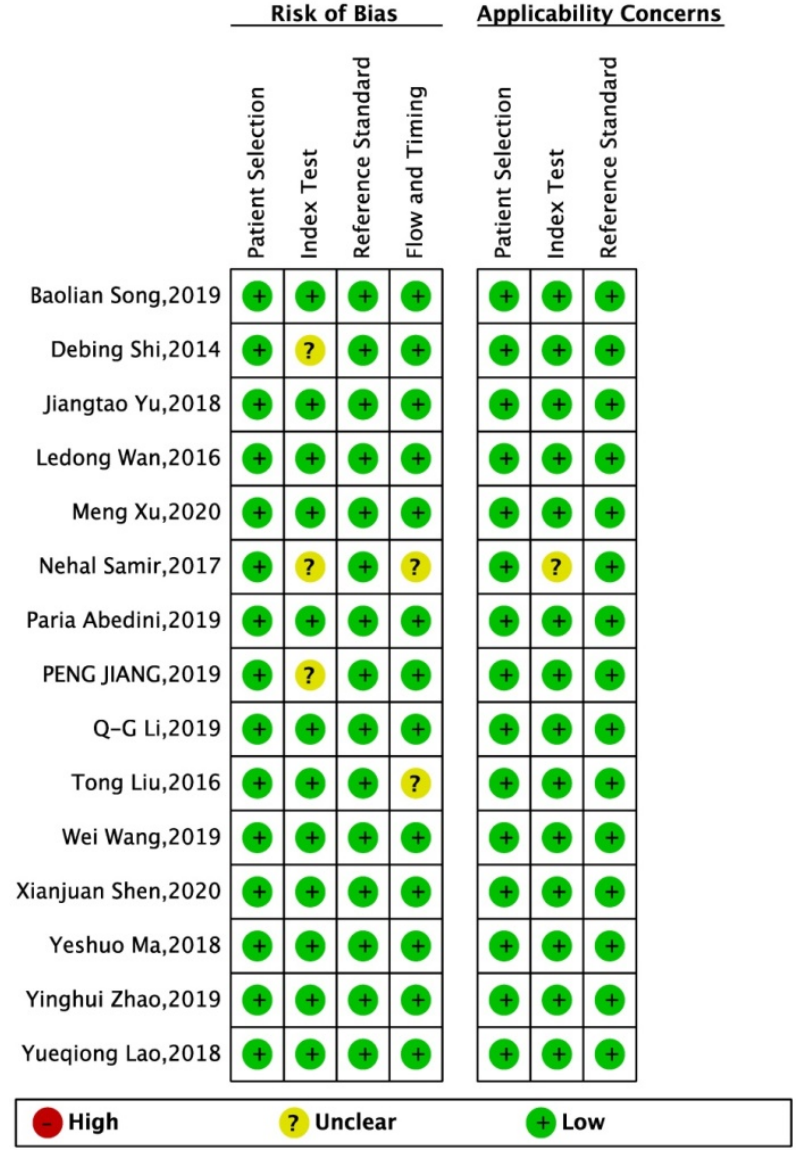

Figure 2. Methodological quality graph.

QUADAS-2 checklist was used to systematically assess the quality of all the included studies. The results indicated that all included studies were of high methodological quality. The results are shown in Figures 2-3.

\section{Meta-analysis of clinicopathological parameters}

The $P$ values between different lncRNAs and clinicopathological parameters in CRC patients of all included studies are summarized in Table 2. Some clinicopathological parameters were not observed or reported in some studies, so the relevant data were not available. Altered expression of lncRNAs were significantly associated with some clinicopathological parameters we collected (tumor size: pooled $P<$ 0.0001; lymphatic metastasis: pooled $P<0.0001$; TNM stage: pooled $P<0.0001$; levels of CEA: pooled $P=$ 0.0007). But, it was not correlated with age (pooled $P=$ 0.5507 ), gender (pooled $P=0.2558$ ), or tumor location (pooled $P=0.7519$ ).

\section{Diagnostic performance}

Forest plots of the pooled sensitivity, pooled specificity, pooled DOR and sROC curve of lncRNAs in diagnosing CRC are shown in Figure 4. The weighted diagnostic parameters of lncRNAs in distinguishing CRC from non-tumor controls are as follows: pooled sensitivity of 0.75 (95\%CI: $0.72-0.77)$, pooled specificity of 0.80 ( $95 \%$ CI: $0.78-0.82)$, pooled PLR of 3.69 (95\%CI: $2.93-4.64$ ), pooled NLR of 0.30 (95\%CI: $0.24-0.37$ ), pooled DOR of 14.20 (95\%CI: 9.27 - 21.75). The AUC of the sROC curve based on summary sensitivity and specificity was 0.86 .

\section{Meta-regression analysis}

Due to the significant heterogeneity between these studies observed in sensitivity and specificity data $\left(I^{2}=87.50 \%\right.$ and $I^{2}=85.80 \%$, respectively $)$, we firstly performed an analysis of diagnostic threshold showed in Table 3 to reveal no threshold effect in the studies (Spearman correlation coefficient: 0.314, $P=$ 0.254). Then we constructed a meta-regression analysis in terms of the specified covariates including sample size, sample types and sample expression of lncRNAs. According to the $P$ value from large to small, "size" and "expression" were eliminated one by one, and meta-regression analysis was performed. The results in Tables 4- 6 showed that the heterogeneity 
might be associated with the sample type (RDOR $=$ accuracy of blood samples was 1.18 times higher than 1.69, 95\%CI: $1.05-2.72, P=0.0339)$. The detection that of non-blood samples, as shown in Table 6.

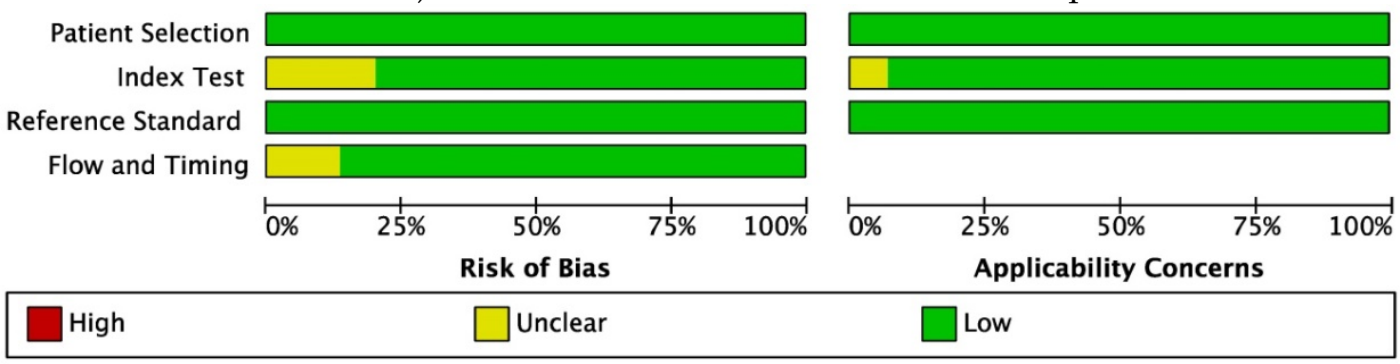

Figure 3. Methodological quality summary.

Table 2. Associations between IncRNAs and clinicopathological parameters in CRC patients

\begin{tabular}{llll}
\hline Clinicopathological parameters & Combined $P$ value & $X^{2}$ value & Enrolled studies \\
\hline Age & 0.55071502 & 26.406427 & 14 \\
Gender & 0.25576268 & 30.290211 & 13 \\
Tumor location & 0.75194819 & 11.883670 & 8 \\
Tumor size $^{*}$ & 0.00000019 & 73.442684 & 11 \\
differentiation $_{\text {Lymphatic metastasis }}^{*}$ & 0.03842262 & 27.284946 & 8 \\
Distal metastasis & 0.00000114 & 88.481443 & 11 \\
TNM stage* & 0.02710721 & 23.073642 & 6 \\
Depth of invasion & 0.00000002 & 83.733916 & 12 \\
CEA level* & 0.01862524 & 18.368803 & 4 \\
CA19-9 level & 0.00073771 & 26.892954 & 4 \\
*P<0.01. & 0.01445876 & 19.075940 & 4
\end{tabular}

Table 3. Analysis of Diagnostic Threshold in weighted regression (inverse variance)

\begin{tabular}{lllll}
\hline Variance & Coefficient & Standard Error & $\mathrm{T}$ & $P$ value \\
\hline $\mathrm{a}$ & 2.693 & 0.237 & 11.383 & 0.0000 \\
$\mathrm{~b}$ & 0.178 & 0.204 & 0.871 & 0.3995 \\
\hline
\end{tabular}

Spearman correlation coefficient: $0.314, p$-value $=0.254$;

Tau-squared estimate $=0.6115$ (convergence is achieved after 6 iterations);

Restricted maximum likelihood estimation (REML);

No. studies $=15$.

Table 4. Meta-regression (inverse variance weights)

\begin{tabular}{llllll}
\hline Variance & Coefficient & Standard Error & $P$ value & RDOC & {$[95 \% \mathrm{CI}]$} \\
\hline Cte. & 2.823 & 1.2690 & 0.0503 & ---- & ---- \\
$\mathrm{S}$ & 0.228 & 0.1873 & 0.2517 & ---- & --- \\
Size & -0.394 & 0.6150 & 0.5366 & 0.67 & $(0.17-2.66)$ \\
Sample & 0.482 & 0.2326 & 0.0651 & 1.62 & $(0.96-2.72)$ \\
Expression & -0.393 & 0.4413 & 0.3951 & 0.67 & $(0.25-1.80)$ \\
\hline & Tau-squared estimate $=0.3808$ (convergence is achieved after 11 iterations); \\
Restricted maximum likelihood estimation (REML); \\
No. studies = 15.
\end{tabular}

Table 5. Meta-regression (remove size)

\begin{tabular}{llllll}
\hline Variance & Coefficient & Standard Error & $P$ value & RDOC & {$[95 \% \mathrm{CI}]$} \\
\hline Cte. & 2.481 & 0.9584 & 0.0293 & --- & --- \\
$\mathrm{S}$ & 0.198 & 0.2100 & 0.3694 & --- & -- \\
Sample & 0.501 & 0.2261 & 0.0488 & 1.65 & $(1.00-2.71)$ \\
Expression & -0.279 & 0.3937 & 0.4937 & 0.76 & $(0.32-1.80)$ \\
\hline
\end{tabular}

Tau-squared estimate $=0.3560$ (convergence is achieved after 10 iterations);

Restricted maximum likelihood estimation (REML);

No. studies $=15$.
Table 6. Meta-regression (remove expression)

\begin{tabular}{llllll}
\hline Variance & Coefficient & Standard Error & $P$ value & RDOC & {$[95 \% \mathrm{CI}]$} \\
\hline Cte. & 1.729 & 0.4298 & 0.0017 & --- & --- \\
$\mathrm{S}$ & 0.268 & 0.1721 & 0.1453 & --- & --- \\
Sample & 0.524 & 0.2187 & $0.0339^{*}$ & 1.69 & $(1.05-2.72)$ \\
\hline \multicolumn{7}{l}{ Tau-squared estimate $=0.3297$ (convergence is achieved after 9 iterations); } \\
Restricted maximum likelihood estimation $($ REML); \\
No. studies $=15$.
\end{tabular}

Therefore, we further analyzed the effect of sample type on diagnosis (Figure 5). A random-effects model was applied because there was significant heterogeneity $\left(I^{2}=63.6 \%\right.$ and $I^{2}=80.6 \%$, respectively $)$. The stratified analysis showed that the performance of blood-based detection was significantly superior to that of tissue-based detection (AUC: 0.8820 vs. 0.8203; pooled DOR: 18.57 vs. 10.47). Blood-based tests were then divided into two categories, plasma-based and serum-based lncRNA testing. The results in Figure 6 revealed that the AUC of serum-based detection was 0.9077, pooled DOR: 26.64, and plasma-based detection was 0.5000 , pooled DOR: 11.80 .

\section{Publication bias}

Deeks' funnel plot asymmetry test was performed to check publication bias in this meta-analysis. The result presented in Fig. 7 showed no evidence of publication bias $(P=0.95)$ existed for diagnostic analyses.

\section{Discussion}

Colorectal cancer is a major cause of cancer mortality worldwide. Early diagnosis greatly increases the chances of successful treatment and improves the cancer outcomes, potentially reducing mortality from cancer [30]. LncRNAs are characterized as a group of endogenous RNAs that have no protein-encoding function [31,32]. Recent studies have suggested that the expression of lncRNAs in CRC might be involved in cancer development, invasion, metastasis, prognosis [33-36]. Statistically significant differences in the expression of lncRNAs between precancerous lesions and early- 
stage cancer have been found in previous studies [37-39]. However, there is no relevant systematic review and meta-analyses focused on the expression of lncRNAs in CRC diagnosis. In this meta-analysis, we systematically analyzed the relationship between the expression of lncRNAs and the early diagnosis of CRC.
A

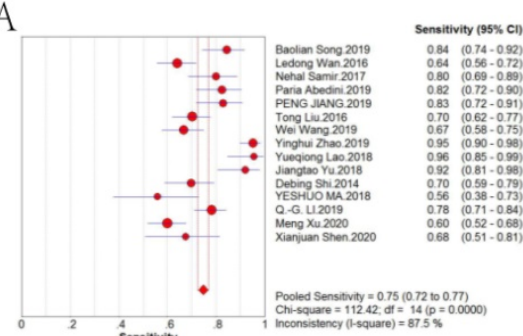

D

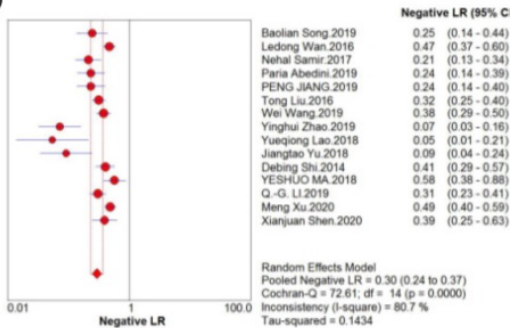

B



E

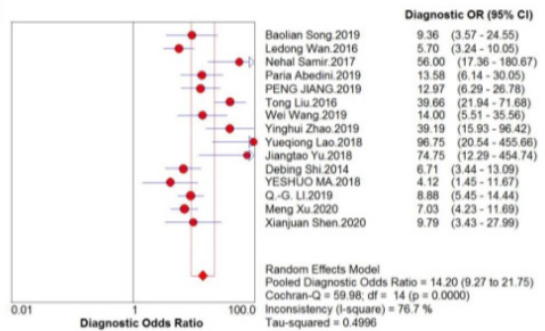

C

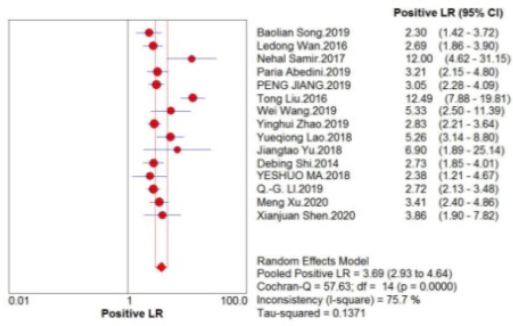

F

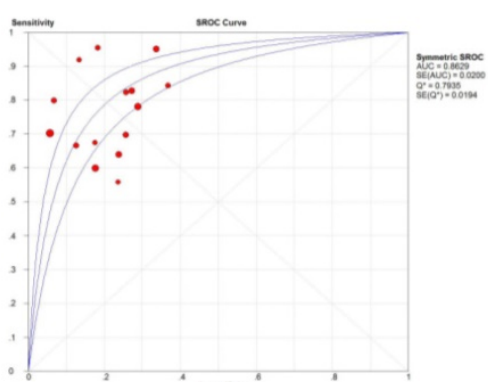

Figure 4. Forest plot of the (A) pooled sensitivity, (B) pooled specificity, (C) pooled PLR, (D) pooled NLR, (E) pooled DOR and (F) sROC curve of IncRNAs for the diagnosis of CRC.

A

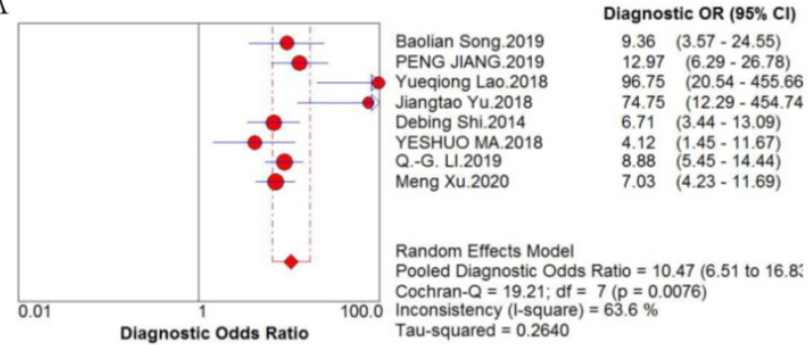

C

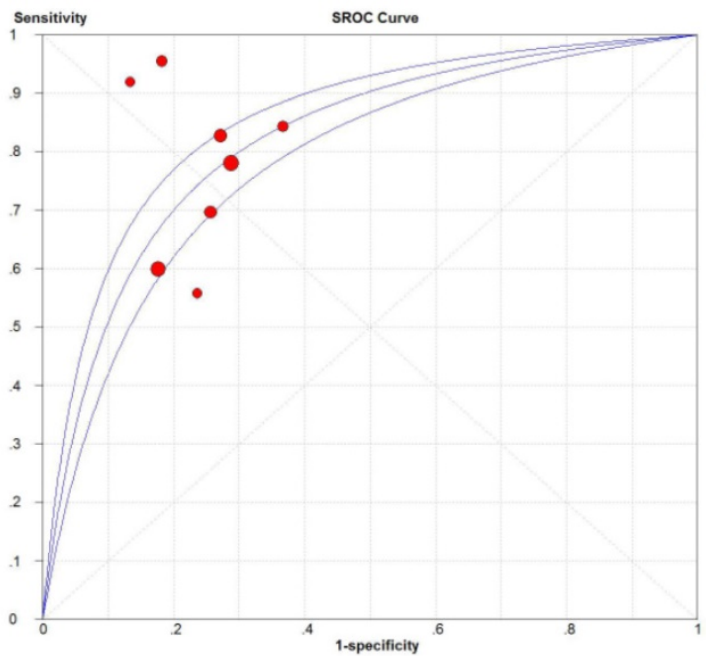

$\mathrm{B}$



$\mathrm{D}$
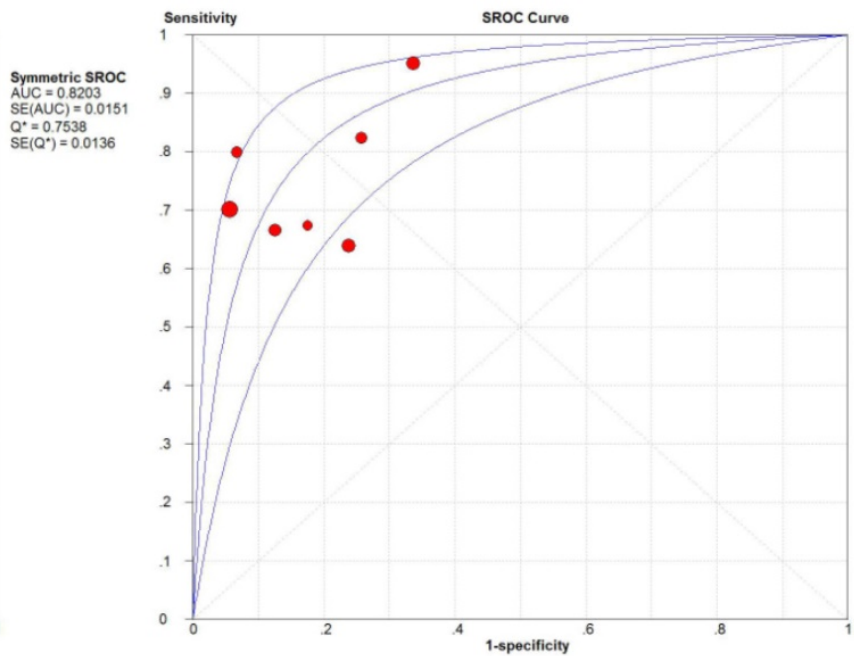

Figure 5. DOR and sROC of (A) tissue-based and (B) blood-based detection of IncRNAs for the diagnosis of CRC. 
A

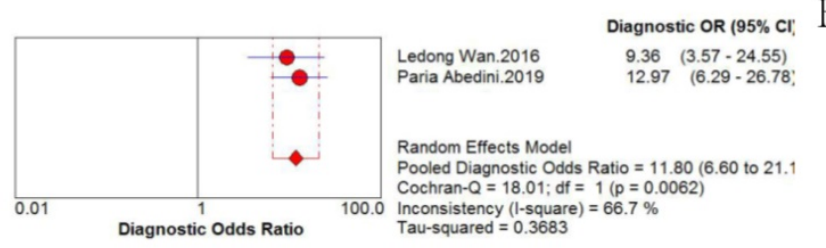

C

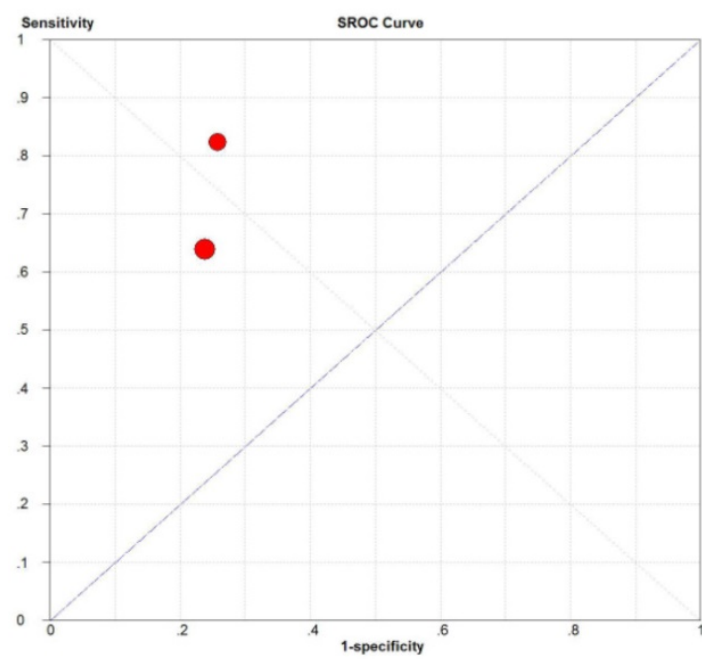



D

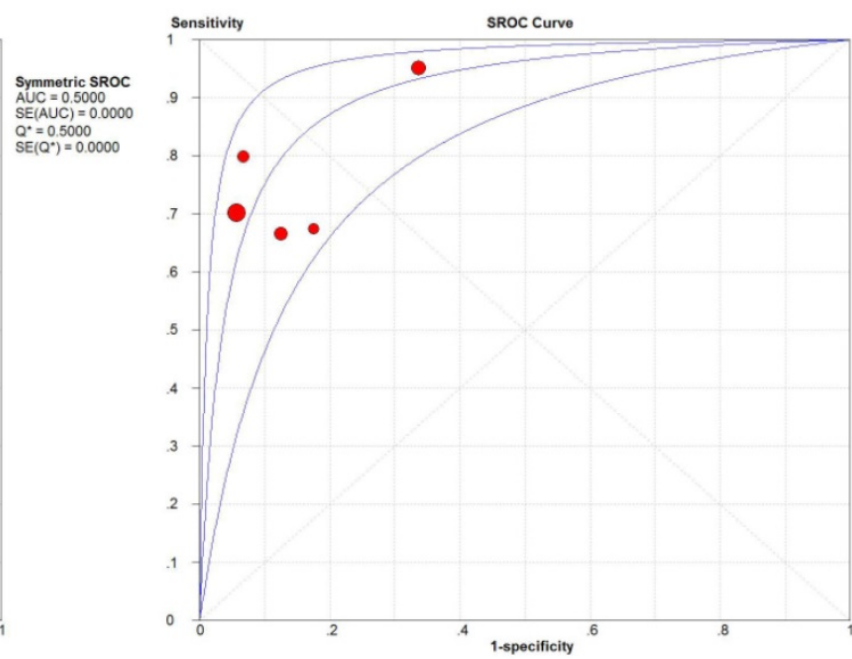

Figure 6. DOR and sROC of (A) plasma-based and (B) serum-based detection of IncRNAs for the diagnosis of CRC.

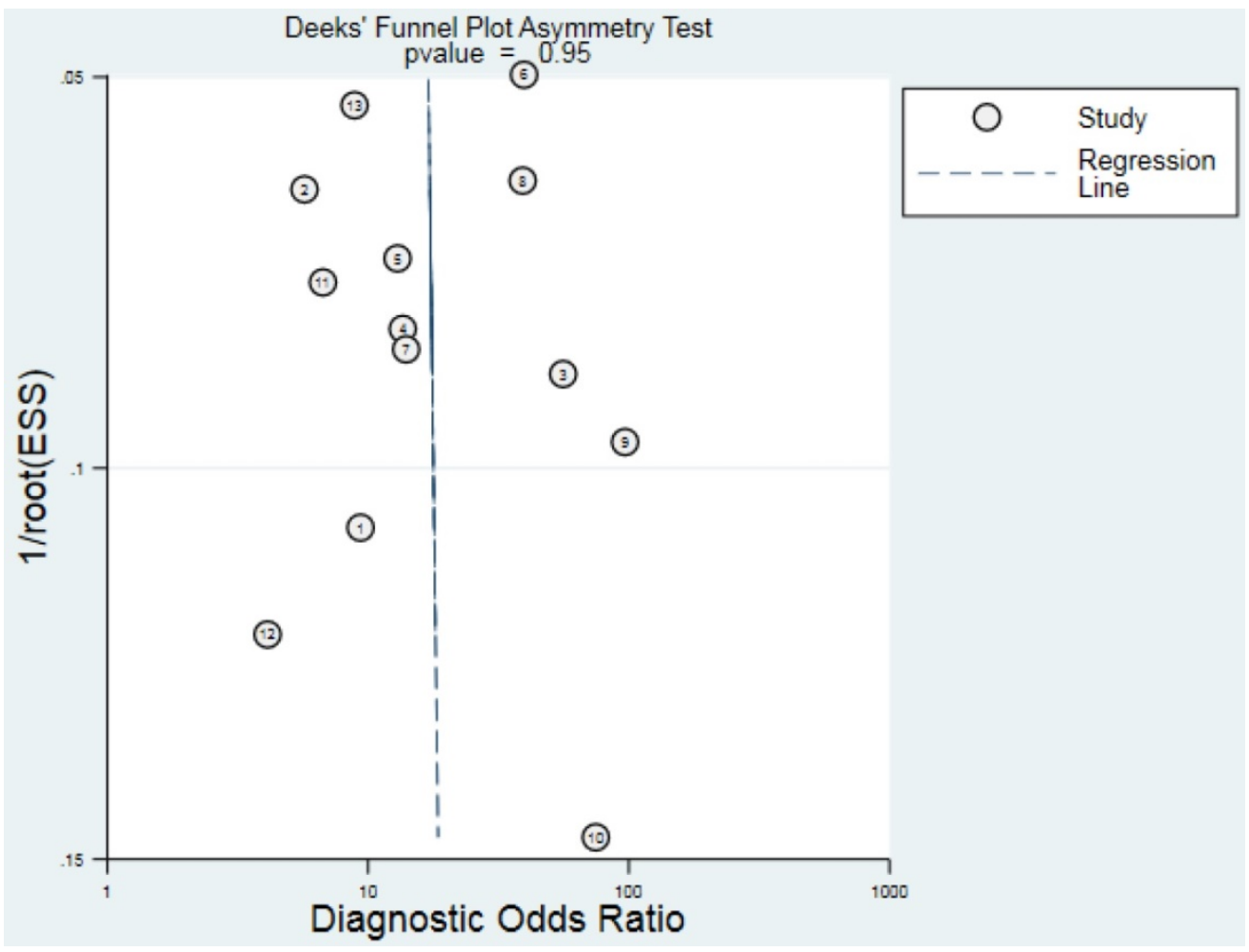

Figure 7. Funnel plot for the assessment of potential publication bias of the diagnostic studies.

The included studies were mainly published between 2016 to 2020 and deemed to be of high quality according to the QUADAS-2. The results of our meta-analysis showed that the expression of 
LncRNAs was significantly associated with tumor size, differentiation, TNM stage, metastasis, levels of CA19-9 and CEA. It indicates that the dysregulated expression of lncRNAs is implicated in the progression of CRC and might act as potential biomarkers for the early diagnosis of CRC.

The ROC curve is a comprehensive index, which reflects the sensitivity and specificity of continuous variables. The pooled AUC of lncRNAs indicated that $86.29 \%$ of randomly chosen CRC patients had higher or lower levels of lncRNAs than normal controls. The pooled DOR is also an important indicator that facilitates formal meta-analysis of studies on diagnostic test performance. In the present study, a pooled DOR of 14.20 (higher than 1.0) was obtained, suggesting that detection of lncRNA is a powerful predictive biomarker for CRC diagnosis.

Fifteen types of lncRNAs with a different expression status in CRC were included in this review. And the meta-regression test further showed that different kind of samples for lncRNA detection was probably the source of heterogeneity $(P=0.0339$, $\mathrm{RDOR}=1.69,95 \% \mathrm{CI}: 1.05-2.72)$. Therefore we conducted subgroup analyses to compare the difference. Stratified analyses based on sample type showed that blood-based lncRNA analysis was of higher diagnostic efficacy than tissue-based analysis (AUC: 0.8820 vs. 0.8203; pooled DOR: 18.57 vs. 10.47). Moreover, serum-based lncRNA testing achieved higher diagnostic efficacy than plasma-based analysis (AUC: 0.9077 vs. 0.5000; pooled DOR: 26.64 vs.11.80), suggesting that serum-based detection is more recommended.

The Deeks' funnel plot asymmetry test didn't reveal obvious publication bias for the diagnostic meta-analyses, suggesting that results from pooled data analysis were reliable. When taking into consideration the stable structures and easy detection, lncRNAs have the potentials to serve as novel, easily attainable biomarkers for the diagnosis of CRC.

Several limitations should be acknowledged in this systematic review and meta-analysis. Firstly, only 15 types of lncRNAs were included and they were analyzed based on different quality control standards, which might have generated potential heterogeneity. Secondly, the small sample size in sub-group analyses led to low statistical power and undermined the strength of the evidence of this systematic review. Thirdly, the majority of patients included in our study were Asians, potential ethnic-related differences in the expression of lncRNAs might restrict the applicability of our findings to other races. Finally, the clinical value of lncRNAs in CRC patients might have been exaggerated because the studies with positive results were more likely to be published than those with negative results.

\section{Conclusion}

In summary, this is the first meta-analysis to evaluate the clinical value of lncRNAs in the diagnosis of colorectal cancer. The results of our meta-analysis reveal that lncRNAs are promising diagnostic biomarkers in patients with CRC. Blood-based lncRNA analysis is of higher diagnostic accuracy than tissue-based testing. Moreover, serum-based lncRNA testing shows higher diagnostic efficacy than plasmabased analysis. However, considering the mentioned limitations above, a larger number of clinical trials are still needed to further verify the findings and confirm the clinical application of lncRNAs in the diagnosis of CRC. Further prospective studies on the role of each specific lncRNA in the early diagnosis of CRC are also warranted in the future.

\section{Acknowledgements}

This study was funded by the Science and Technology Development Fund, Macau SAR (130/2017/A3, 0099/2018/A3, 0096/2018/A3), the National Natural Science Foundation of China (grant No. 81874380, 81672932 and 81730108), the Zhejiang Provincial Natural Science Foundation of China for Distinguished Young Scholars (Grant No. LR18H160001), the Zhejiang province science and technology project of TCM (grant No. 2019ZZ016) and the Key Project of Hangzhou Ministry of Science and Technology (grant No. 20162013A07, 20142013A63).

\section{Author Contributions}

Qibiao $\mathrm{Wu}$, Xinbing Sui and Elaine Lai-Han Leung designed this study. Bi Chen and Ruonan Zhang participated in study selection and data extraction. Bi Chen, Xingxing Fan, Jue Wang, Cong $\mathrm{Xu}$, Bo An, Jing Wang, and Qiao Wang performed the statistical analysis. Bi Chen, Qibiao $\mathrm{Wu}$, Xinbing Sui and Elaine Lai-Han Leung wrote and revised the manuscript. All authors approved the submission of the article.

\section{Competing Interests}

The authors have declared that no competing interest exists.

\section{References}

1. Bray F, Ferlay J, Soerjomataram I, Siegel RL, Torre LA, Jemal A. Global cancer statistics 2018: GLOBOCAN estimates of incidence and mortality worldwide for 36 cancers in 185 countries. CA Cancer J Clin. 2018; 68: 394-424.

2. Zheng RS, Sun KX, Zhang SW, Zeng HM, Zou XN, Chen R, et al. [Report of cancer epidemiology in China, 2015]. Zhonghua Zhong Liu Za Zhi. 2019; 41: 19-28.

3. Weng W, Liu N, Toiyama Y, Kusunoki M, Nagasaka T, Fujiwara T, et al. Novel evidence for a PIWI-interacting RNA (piRNA) as an oncogenic mediator of 
disease progression, and a potential prognostic biomarker in colorectal cancer. Mol Cancer. 2018; 17: 16-28.

4. Zheng C, Jiang F, Lin H, Li S. Clinical characteristics and prognosis of different primary tumor location in colorectal cancer: a population-based cohort study. Clin Transl Oncol. 2019; 21: 1524-31.

5. Li CF, Li YC, Wang Y, Sun LB. The Effect of LncRNA H19/miR-194-5p Axis on the Epithelial-Mesenchymal Transition of Colorectal Adenocarcinoma. Cellular physiology and biochemistry : international journal of experimental cellular physiology, biochemistry, and pharmacology. 2018; 50: 196-213.

6. Xie H, Ma B, Gao Q, Zhan H, Liu Y, Chen Z, et al. Long non-coding RNA CRNDE in cancer prognosis: Review and meta-analysis. Clinica chimica acta; international journal of clinical chemistry. 2018; 485: 262-71.

7. Jiang $\mathrm{P}, \mathrm{Han} \mathrm{XT}$, Zheng YN, Sui JC, Bi WP. Long non-coding RNA NKILA serves as a biomarker in the early diagnosis and prognosis of patients with colorectal cancer. Oncology Letters. 2019; 18: 2109-17.

8. Lipovich L, Johnson R, Lin CY. MacroRNA underdogs in a microRNA world: evolutionary, regulatory, and biomedical significance of mammalian long non-protein-coding RNA. Biochim Biophys Acta. 2010; 1799: 597-615.

9. Wang W, Xie Y, Chen F, Liu X, Zhong LL, Wang HQ, et al. LncRNA MEG3 acts a biomarker and regulates cell functions by targeting ADAR1 in colorectal cancer. World journal of gastroenterology. 2019; 25: 3972-84.

10. Abedini P, Fattahi A, Agah S, Talebi A, Beygi AH, Amini SM, et al. Expression analysis of circulating plasma long noncoding RNAs in colorectal cancer: The relevance of lncRNAs ATB and CCAT1 as potential clinical hallmarks. Journal of Cellular Physiology. 2019; 234: 22028-33.

11. Dai M, Chen X, Mo S, Li J, Huang Z, Huang S, et al. Meta-signature LncRNAs serve as novel biomarkers for colorectal cancer: integrated bioinformatics analysis, experimental validation and diagnostic evaluation. Sci Rep. 2017; 7: 46572-80

12. Wan L, Kong J, Tang J, Wu Y, Xu E, Lai M, et al. HOTAIRM1 as a potential biomarker for diagnosis of colorectal cancer functions the role in the tumour suppressor. Journal of cellular and molecular medicine. 2016; 20: 2036-44.

13. Liu T, Zhang X, Gao S, Jing F, Yang Y, Du L, et al. Exosomal long noncoding RNA CRNDE-h as a novel serum-based biomarker for diagnosis and prognosis of colorectal cancer. Oncotarget. 2016; 7: 85551-63.

14. Zhao Y, Du T, Du L, Li P, Li J, Duan W, et al. Long noncoding RNA LINC02418 regulates MELK expression by acting as a ceRNA and may serve as a diagnostic marker for colorectal cancer. Cell Death Dis. 2019; 10: 568-81.

15. Penny F. Whiting PAWSR, PhD; Marie E. Westwood, PhD; Susan Mallett, PhD; Jonathan J. Deeks, PhD; Johannes B. Reitsma, MD, PhD; Mariska M.G. Leeflang, PhD; Jonathan A.C. Sterne, PhD; Patrick M.M. Bossuyt, PhD; and the QUADAS-2 Group*. QUADAS-2: A Revised Tool for the Quality Assessment of Diagnostic Accuracy Studies. Annals of Internal Medicine. 2011; 155: 529-36.

16. Zamora J, Abraira V, Muriel A, Khan K, Coomarasamy A. Meta-DiSc: a software for meta-analysis of test accuracy data. BMC Med Res Methodol. 2006; 6: 31-43.

17. Chen H, Yao X, Li T, Lam CW, Zhang H, Wang J, et al. Compound Kushen injection plus platinum-based chemotherapy for stage IIIB/IV non-small cell lung cancer: A protocol for meta-analysis of randomized clinical trials following the PRISMA guidelines. Medicine (Baltimore). 2019; 98: 18552-6.

18. Chang $\mathrm{H}$, Wang GN, Tao YL. The expression of long noncoding RNA CRCAL-3 in colorectal cancer and its impacts on cell proliferation and migration. Journal of cellular biochemistry. 2019; 120: 15369-77.

19. Wu Q, Lai-Han Leung E. Commentary on Ge et al. "Insomnia and risk of mortality from all-cause, cardiovascular disease, and cancer: Systematic review and meta-analysis of prospective cohort studies". Sleep Med Rev. 2020; 50: $101257-68$

20. Deeks JJ, Macaskill P, Irwig L. The performance of tests of publication bias and other sample size effects in systematic reviews of diagnostic test accuracy was assessed. J Clin Epidemiol. 2005; 58: 882-93.

21. Song B, Jia Y. High expression of Plnc RNA-1 promotes the progression of colorectal cancer. Journal of BUON : official journal of the Balkan Union of Oncology. 2019; 24: 99-105.

22. Hu D, Zhan Y, Zhu K, Bai M, Han J, Si Y, et al. Plasma Exosomal Long Non-Coding RNAs Serve as Biomarkers for Early Detection of Colorectal Cancer. Cellular physiology and biochemistry : international journal of experimental cellular physiology, biochemistry, and pharmacology. 2018; 51: 2704-15.

23. Lao $\mathrm{Y}$, $\mathrm{Li} \mathrm{O}, \mathrm{Li} \mathrm{N}$, Liu $\mathrm{H}$, Liu $\mathrm{K}$, Jiang $\mathrm{G}$, et al Long noncoding RNA ENST00000455974 plays an oncogenic role through up-regulating JAG2 in human DNA mismatch repair-proficient colon cancer. Biochemical and biophysical research communications. 2019; 508: 339-47.

24. Shen X, Xue Y, Cong H, Wang X, Fan Z, Cui X, et al. Circulating lncRNA DANCR as a potential auxillary biomarker for the diagnosis and prognostic prediction of colorectal cancer. Bioscience Reports. 2020; 40: 12-9.

25. Xu M, Guo X, Wang RD, Zhang ZH, Jia YM, Sun X. Long non-coding RNA HANR as a biomarker for the diagnosis and prognosis of colorectal cancer. Medicine. 2020; 99: 23-8.

26. Zhao W, Song M, Zhang J, Kuerban M, Wang $\mathrm{H}$. Combined identification of long non-coding RNA CCAT1 and HOTAIR in serum as an effective screening for colorectal carcinoma. International journal of clinical and experimental pathology. 2015; 8: 14131-40.

27. Yan H, Zheng G, Qu J, Liu Y, Huang X, Zhang E, et al. Identification of key candidate genes and pathways in multiple myeloma by integrated bioinformatics analysis. J Cell Physiol. 2019; 234: 23785-97.
28. Samir N, Matboli M, El-Tayeb H, El-Tawdi A, Hassan MK, Waly A, et al. Competing endogenous RNA network crosstalk reveals novel molecular markers in colorectal cancer. Journal of cellular biochemistry. 2018; 119: 6869-81.

29. Shi D, Zheng H, Zhuo C, Peng J, Li D, Xu Y, et al. Low expression of novel IncRNA RP11-462C24.1 suggests a biomarker of poor prognosis in colorectal cancer. Med Oncol. 2014; 31: 31-8.

30. Roncucci L, Mariani F. Prevention of colorectal cancer: How many tools do we have in our basket? Eur J Intern Med. 2015; 26: 752-6.

31. Gini A, Jansen EEL, Zielonke N, Meester RGS, Senore C, Anttila A, et al. Impact of colorectal cancer screening on cancer-specific mortality in Europe: A systematic review. Eur J Cancer. 2020; 127: 224-35.

32. Lv X, Li Y, Li Y, Li H, Zhou L, Wang B, et al. FAL1: A critical oncogenic long non-coding RNA in human cancers. Life Sci. 2019; 236: 116918-25.

33. Luo J, Qu J, Wu DK, Lu ZL, Sun YS, Qu Q. Long non-coding RNAs: a rising biotarget in colorectal cancer. Oncotarget. 2017; 8: 22187-202.

34. Yang X, Meng T. Long Noncoding RNA in Preeclampsia: Transcriptional Noise or Innovative Indicators? BioMed Research International. 2019; 2019. $1-7$.

35. Yang DD, Chen ZH, Wang DS, Yu HE, Lu JH, Xu RH, et al. Prognostic value of the serum apolipoprotein $\mathrm{B}$ to apolipoprotein A-I ratio in metastatic colorectal cancer patients. J Cancer. 2020; 11: 1063-74.

36. Janaki Ramaiah M, Divyapriya K, Kartik Kumar S, Rajesh Y. Drug-induced modifications and modulations of microRNAs and long non-coding RNAs for future therapy against Glioblastoma Multiforme. Gene. 2020; 723: 144126-35.

37. Xu W, Zhou G, Wang H, Liu Y, Chen B, Chen W, et al. Circulating IncRNA SNHG11 as a novel biomarker for early diagnosis and prognosis of colorectal cancer. Int J Cancer. 2020; 146: 2901-12.

38. Sun L, Liu X, Pan B, Hu X, Zhu Y, Su Y, et al. Serum exosomal miR-122 as a potential diagnostic and prognostic biomarker of colorectal cancer with liver metastasis. J Cancer. 2020; 11: 630-7.

39. Zhou Y, Wen X, Zhang Q, Kou W. Expression of IncRNA SNHG16 in colorectal cancer tissues and cells and its regu-lationon expression of GPAM in colon cancer cells. Chinese Journal of Cancer Biotherapy. 2019; 26: 58-66. 